\title{
A PROPERTY OF POLYNOMIALS WHOSE ROOTS ARE REAL
}

\author{
By G. S. Le Beav.
}

[Read March 11th, 1920.]

1. Let $a_{1}, a_{2}, a_{3}, \ldots, a_{i}$, be the roots, supposed real, unequal, and in ascending order of magnitude, of a polynomial

$$
f(x) \equiv x^{n}-a_{1} x^{n-1}+a_{2} x^{n-2}-\ldots,
$$

and let $\beta_{1}, \beta_{2}, \ldots, \beta_{n-1}$, be the roots, also real and in ascending order of magnitude, of

$$
\phi(x) \equiv x^{n-1}-b_{1} x^{n-2}+\ldots ;
$$

further, let the roots of $\phi(x)$ separate those of $f(x)$, so that

$$
a_{1}<\beta_{1} \leqslant \alpha_{2} \leqslant \beta_{2} \leqslant \cdots \leqslant \beta_{n-1}<\alpha_{n} .
$$

Let $f(x)$ be divided by $\phi(x)$ and let the quotient be $x-\gamma$ and the remainder $-A \psi(x)$, where

$$
\psi(x) \equiv x^{n-2}-c_{1} x^{n-3}+\ldots
$$

Then denoting the ronts of $\psi(x)$ by $\delta_{1}, \delta_{2}, \ldots, \delta_{n-2}$, it is immediately seen that the $\delta$ 's are all real and that they separate the $\beta$ 's, and further that $\gamma$ and the $\delta$ 's separate the $a$ 's. We can thus take $\gamma$ and the $\delta$ 's together as a new set of $\beta$ 's : that is, we take, as a new $\phi(x)$, the polynomial

$$
(x-\gamma)\left(x-\delta_{1}\right)\left(x-\delta_{2}\right) \ldots\left(x-\delta_{n-2}\right)
$$

and divide $f(x)$ by it as before, obtaining a new $\gamma$ and new $\delta$ 's, which separate the $\alpha$ 's as before, and so on, continuing in this way indefinitely. We shall show that $\delta_{1}, \delta_{2}, \ldots, \delta_{n-2}$ tend to the limits $\alpha_{2}, a_{3}, \ldots, a_{n-1}$.

2. Considering any stage of the process, let the greatest value of

$$
\left|\beta_{s}-\frac{1}{2}\left(\alpha_{1}+\alpha_{n}\right)\right|(s=1,2,8, \ldots, n-1)
$$

be denotel by $g$. Then since

$$
\beta_{1} \leqslant \delta_{1} \leqslant \beta_{2} \leqslant \delta_{2} \leqslant \cdots \leqslant \delta_{n-2} \leqslant \beta_{n-1},
$$


it follows that

$$
\left|\hat{o}_{s}-\frac{1}{2}\left(\alpha_{1}+\alpha_{n}\right)\right| \leqslant g \quad(s=1,2,3, \ldots, n-2) .
$$

Also

$$
\gamma=\sum_{s=1}^{n} \alpha_{s}-\sum_{s=1}^{n-1} \beta_{s}
$$

and since

$$
\alpha_{1} \leqslant \beta_{1} \leqslant a_{2} \leqslant \beta_{2} \leqslant \ldots \leqslant \beta_{n-1} \leqslant a_{n},
$$

we get

$$
\begin{aligned}
g \geqslant \frac{1}{2}\left(\alpha_{1}+\alpha_{n}\right)-\beta_{1} & \geqslant \gamma-\frac{1}{2}\left(a_{1}+a_{n}\right) \\
& \geqslant \frac{1}{2}\left(\alpha_{1}+a_{n}\right)-\beta_{n-1} \\
& \geqslant-g .
\end{aligned}
$$

Hence

$$
\left|\gamma-\frac{1}{2}\left(\alpha_{1}+a_{n}\right)\right| \leqslant g \text {. }
$$

From (2) and (4) we get that if $g^{\prime}$ is the greatest value of $\left|\beta_{s}-\frac{1}{2}\left(\alpha_{1}+\alpha_{n}\right)\right|$ for the next stage of the process,

$$
g^{\prime} \leqslant g .
$$

Thus at no stage does $g$ increase, and, since it is essentially positive, it tends to a definite limit.

3. Again, from the equation

$$
f^{\prime}(x)=(x-\gamma) \phi(c)-A \psi(x),
$$

we get, on equating coefficients of $x^{n-2}$,

$$
\begin{aligned}
A= & \Sigma \beta_{1} \beta_{2}-\Sigma \alpha_{1} \alpha_{2}+\gamma \Sigma \beta_{1} \\
= & \Sigma \beta_{1} \beta_{2}-\sum \alpha_{1} \alpha_{2}+\left(\Sigma \alpha_{1}-\Sigma \beta_{1}\right) \Sigma \beta_{1} \\
= & \left(a_{n}-\beta_{n-1}\right) \sum_{s=1}^{n-1}\left(\beta_{s}-\alpha_{s}\right)+\left(\alpha_{n-1}-\beta_{n-2}\right) \sum_{s=1}^{n-2}\left(\beta_{s}-a_{s}\right) \\
& \quad+\ldots+\left(\alpha_{2}-\beta_{1}\right)\left(\beta_{1}-\alpha_{1}\right),
\end{aligned}
$$

so that it follows from (3) that $A$ cannot be negative.

If we denote the value of $A$ at the next stage by $A^{\prime}$, we have

$$
A^{\prime}=\left(\sum \delta_{1} \delta_{2}+\gamma \Sigma \delta_{1}\right)-\Sigma \alpha_{1} \alpha_{2}+\left(\gamma+\Sigma \delta_{1}\right)\left(\sum \alpha_{1}-\gamma-\Sigma \delta_{1}\right),
$$

since $\gamma$ and the $\delta$ 's constitute the new $\beta$ 's. 
Thue $A^{\prime}=\Sigma \delta_{1} \delta_{2}+\gamma \Sigma \delta_{1}-\Sigma a_{1} a_{2}+\left(\gamma+\Sigma \delta_{1}\right)\left(\Sigma \beta_{1}-\Sigma \delta_{1}\right)$

$$
=\Sigma \delta_{1} \delta_{2}-\Sigma \alpha_{1} \alpha_{2}+\gamma \Sigma \beta_{1}+\left(\Sigma \beta_{1}-\Sigma \delta_{1}\right) \Sigma \delta_{1} \text {. }
$$

From (5) and (6), we get

$$
\begin{aligned}
A^{\prime}-A= & \Sigma \delta_{1} \delta_{2}-\Sigma \beta_{1} \beta_{2}+\left(\Sigma \beta_{1}-\Sigma \delta_{1}\right) \Sigma \delta_{1} \\
= & \left(\beta_{n-1}-\delta_{n-2}\right) \sum_{s=1}^{n-2}\left(\delta_{s}-\beta_{s}\right)+\left(\beta_{n-2}-\delta_{n-3}\right) \sum_{s=1}^{n-3}\left(\delta_{s}-\beta_{s}\right) \\
& +\ldots+\left(\beta_{2}-\delta_{1}\right)\left(\delta_{1}-\beta_{1}\right),
\end{aligned}
$$

so that, from (1), it follows that

$$
A^{\prime} \geqslant A \text {. }
$$

Since all the $\beta$ 's lie between assignable limits, it is obvious that $A$ cannot exceed an assignuble value. Hence $A$ tends to a definite limit.

4. It follows trom this result that, $\epsilon$ being a given arbitrarily small positive quantity, from and after a certain stage the expression on the right-hand side of (7) will be less than $\epsilon^{2}$. Since all the products of differences of which this expression is composed are positive, and the expression contrins the product $\left(\beta_{s+1}-\delta_{s}\right)\left(\delta_{s}-\beta_{s}\right)$, we shall then have

$$
\left(\beta_{s+1}-\delta_{g}\right)\left(\delta_{s}-\beta_{s}\right)<\epsilon^{2} \quad(s=1,2,3, \ldots, n-2),
$$

and hence either $\quad \beta_{i+1}-\delta_{s}<\epsilon$ or $\delta_{s}-\beta_{s}<\epsilon$.

Writing $x=\delta_{s}$ in the identity

$$
f(x)=(x-\gamma) \phi(x)-A \psi(x),
$$

we get

$$
f\left(\delta_{s}\right)=\left(\delta_{s}-\gamma\right) \phi\left(\delta_{s}\right),
$$

and it follows from (8) that

$$
\left|f\left(\delta_{s}\right)\right|<\epsilon\left(\alpha_{n}-\alpha_{1}\right)^{n-1}
$$

Hence from and after a certain stage, $\delta_{s}$ differs from some one of the $a^{\prime}$ 's by less than an arbitrarily small $\epsilon_{1}$. If $\epsilon_{1}$ is sufficiently small, this one of the a's cannot be either $\alpha_{1}$ or $\alpha_{n}$. For, if the initial values of $\beta_{1}-\alpha_{1}$ and $\alpha_{n}-\beta_{n-1}$ both exceed $h$, say, it follows from the result of $\S 2$ that, at every stage of the process, the difference between any $\delta$ and either $\alpha_{1}$ or $\alpha_{n}$ also exceeds $h$. 
We thus arrive at the result that, $\epsilon$ being given and arbitrarily small, from and after a certain stage in the process, every $\delta$ will differ from some one of $a_{2}, a_{3}, \ldots, a_{n-1}$, by a quantity less than $\epsilon$.

5. We will next prove that if the process is carried sufficiently far, and if $\epsilon$ is sufficiently small, it is impossible that two $\delta$ 's should differ from the same $\alpha$ by quantities less than $\epsilon$.

For suppose that $2 \epsilon$ is less than the difference between any two consecutive $\alpha^{\prime}$ s, and that $\delta$ and $\delta^{\prime}$ both differ from $\alpha_{r}$ by less than $\epsilon$. Since the interval between two consecutive $\alpha$ 's cannot contain more than one $\delta$, it follows that $\delta$ and $\delta^{\prime}$ are consecutive $\delta^{\prime}$ s. Let $\beta, \beta^{\prime}, \beta^{\prime \prime}$, be the consecutive $\beta$ 's which they separate. Then we shall bave either

or

$$
\text { (i) } \beta<a_{r-1}<\delta<\beta^{\prime}<a_{r}<\delta^{\prime}<\beta^{\prime \prime} \text {, }
$$$$
\text { (ii) } \beta<\delta<a^{\prime}<\beta^{\prime}<\delta^{\prime}<a_{r+1}<\beta^{\prime \prime} \text {. }
$$

We can suppose the process carried so far that the expression on the right-hand side of (7) is less than either $\frac{1}{2} \epsilon\left(a_{r}-a_{r-1}\right)$ or $\frac{1}{2} \epsilon\left(\alpha_{r+1}-\alpha_{r}\right)$. Hence $\left(\beta^{\prime \prime}-\delta^{\prime}\right)(\delta-\beta)$ is less than either of these quantities.

Now, in case (i),

$$
\delta-\beta>\frac{1}{2}\left(u_{r}-a_{r-1}\right), \quad \beta^{\prime \prime}-\delta^{\prime}<\epsilon, \quad \beta^{\prime \prime}-a_{r}<2 \epsilon,
$$

and in case (ii),

$$
\beta^{\prime \prime}-\delta^{\prime}>\frac{1}{2}\left(a_{r+1}-\alpha_{r}\right), \quad \delta-\beta<\epsilon, \quad \alpha_{r}-\beta<2 \epsilon .
$$

'Thus in either case we shall have two of the $\delta$ 's and two of the $\beta$ 's all differing from $\alpha_{r}$ by less than $2 \epsilon$.

Hence from the equation

$$
f(x)=(x-\gamma) \phi(x)-A \psi(x),
$$

we get, observing that the right-band side must be divisible by $x-a_{\text {, }}$,

$$
\left(x-a_{1}\right)\left(x-a_{2}\right) \ldots\left(x-a_{n}\right)=\left(x-\alpha_{r}\right)^{2} F(x)+\epsilon\left(x-a_{r}\right) G(x),
$$

where $\left|G\left(a_{r}\right)\right|$ cannot exceed an assiguable fixed value, independent of $\epsilon$.

Dividing by $x-a_{r}$ and putting $x=a_{r}$, we get

$$
f^{\prime}\left(a_{r}\right)=\epsilon G\left(a_{r}\right),
$$

which is impossible if $\epsilon$ is sufficiently small. This proves the result required. 
6. If, then, $\epsilon_{1}$ is sufficiently small, and we choose any $\epsilon$ less than $\epsilon_{1}$, we shall have, from and after a certain stage, every $\delta$ differing from one of $a_{2}, \alpha_{3}, \ldots, u_{n-1}$ by less than $\epsilon$, and no two $\delta$ 's differing from the same a by less than $\epsilon$. Thus we shall have

$$
\left|\delta_{1}-\alpha_{3}\right|<\epsilon, \quad\left|\delta_{2}-\alpha_{3}\right|<\epsilon, \quad \ldots, \quad\left|\delta_{n-2}-\alpha_{n-1}\right|<\epsilon .
$$

Thus $\delta_{1}, \delta_{2}, \ldots, \delta_{n-2}$ tend to the limits $a_{2}, \alpha_{3}, \ldots, \alpha_{n-1}$.

If $\gamma$ and $\gamma^{\prime}$ are the values of $\gamma$ obtained in two consecutive stages of the operation, it immediately follows from this result. combined with the equation

$$
\gamma=\sum_{s=1}^{n} \alpha_{s}-\sum_{s=1}^{n-1} \beta_{s},
$$

that $\gamma+\gamma^{\prime}$ tends to the limit $\alpha_{1}+\alpha_{n}$. Hence the alternate values of $\gamma$ tend to limits $l, l^{\prime}$, such that $l+l^{\prime}=a_{1}+a_{n}$, and we evidently have that if $L$ is the limiting value of $A$,

$$
\left(x-\alpha_{1}\right)\left(x-\alpha_{n}\right)=(x-l)\left(x-l^{\prime}\right)-L,
$$

so that

$$
L=l l^{\prime}-\alpha_{1} \alpha_{\prime \prime} .
$$

The values of $l, l^{\prime}, L$, depend on the initial choice of $\beta_{1}, \beta_{2}, \ldots, \beta_{n-}$

7. We have

$$
L=\left(l-a_{1}\right)\left(\alpha_{n}-l\right)=\left(l-a_{1}\right)\left(l^{\prime}-a_{1}\right)=\left(u_{n}-l\right)\left(a_{n}-l^{\prime}\right) .
$$

It may be observed that

$$
\left|\left(a_{r}-l\right)\left(a_{r}-l^{\prime}\right)\right| \leqslant\left(l-\alpha_{1}\right)\left(l^{\prime}-a_{1}\right) \quad(r=2,3, \ldots, n-1) .
$$

For suppose, if possible, that

where $\lambda$ is positive.

$$
\left|\frac{\left(a_{r}-l\right)\left(a_{r}-l^{\prime}\right)}{\left(a_{1}-l\right)\left(a_{1}-l^{\prime}\right)}\right|=1+\lambda,
$$

From the equation

$$
\begin{aligned}
&\left(x-\alpha_{1}\right)\left(x-\alpha_{2}\right) \ldots\left(x-\alpha_{n}\right) \\
& \quad=\left(x-\gamma^{\prime}\right)(x-\gamma)\left(x-\delta_{1}\right) \ldots\left(x-\delta_{n-2}\right)-A\left(x-\delta_{1}^{\prime}\right)\left(x-\delta_{:}^{\prime}\right) \ldots\left(x-\delta_{n-2}^{\prime}\right),
\end{aligned}
$$

we get, putting $x=a_{r}$,

$$
\frac{a_{r}-\delta_{r-1}^{\prime}}{a_{r}-\delta_{r-1}}=\frac{\left(\alpha_{r}-\gamma^{\prime}\right)\left(a_{r}-\gamma\right)}{A} \prod_{s}\left(\frac{a_{r}-\delta_{s}}{a_{r}-\delta_{s}^{\prime}}\right),
$$


where the product is taken for every value of $s$ from 1 to $n-2$ inclusive, except $r-1$. Since $\delta_{1}, \delta_{2}, \ldots$ tend to limits $\alpha_{3}, a_{3}, \ldots$, it follows that this product tends to the limit unity. Also

$$
\operatorname{Lim}\left|\frac{\left(\alpha_{r}-\gamma^{\prime}\right)\left(\alpha_{r}-\gamma\right)}{A}\right|=1+\lambda .
$$

Hence from and after a certain stage, we shall have

$$
\left|\frac{\left(a_{r}-\gamma^{\prime}\right)\left(a_{r}-\gamma\right)}{A}\right|>1+\frac{1}{2} \lambda, \text { and } \mid \text { I.I }\left(\frac{a_{r}-\delta_{i}}{\alpha_{r}-\delta_{s}^{\prime}}\right) \mid>\frac{1}{1+\frac{1}{2} \lambda},
$$

so that from and after this stage

$$
\left|\frac{a_{r}-\delta_{r-1}^{\prime}}{a_{r}-\delta_{r-1}}\right|>1
$$

which is impossible, since $\operatorname{Lim} \dot{\delta}_{r-1}=\alpha_{r}$.

8. It has been supposed that the roots of $f(x)$ are unequal, but we may evidently dispense with this condition, for if $f(x)$ contains a factor $(x-\alpha)^{r}$, we must suppose the original $\phi(x)$ to contain a factor $(x-a)^{r-1}$, so that $\psi(x)$ also contains a factor $(x-\alpha)^{r-1}$, and so on. In fact, the polynomial $\psi(x)$ obtained at any stage is $(x-\alpha)^{r-1} \psi_{1}(x)$, where $\psi_{1}(x)$ is the polynomial obtained at the corresponding stage of the process of repeated division of $f(x) /(x-\alpha)^{r-1}$. The theorem thus holds in all cases when the roots of $f(x)$ are real.

If we divide $f(x)$ by the successive polynomials $\psi(x)$, we get a succession of quadratics, whose roots tend to the limits $\alpha_{1}$ and $\alpha_{n}$. Taking a polynomial $\psi(x)$, whose roots are sufficiently close to $a_{2}, \alpha_{3}, \ldots, a_{n-1}$, we can apply the same process to it, and in this way we can obtain a quadratic whose roots are as close as we please to $\alpha_{2}$ and $a_{n-1}$, and so on. We can thus obtain a set of equations, which are all quadratic if $n$ be even, or one linear and the rest quadratic if $n$ be odd, whose roots approximate as closely as we please to $\alpha_{1}$ and $\alpha_{n}, \alpha_{2}$ and $\alpha_{n-1}, \alpha_{3}$ and $\alpha_{n-2}$, \&c.

In particular, we can take, for the initial $\phi(x)$, the first derived $f^{\prime}(x)$ of $f^{\prime}(x)$. In this case, the coefficients in the quadratic equations are rational functions of the coefficients of $f(x)$. The process can thus be applied to an algebraic function $y$ defined by an equation $f(y, x)=0$, and we get the following result:- If for all real values of $x$ in the range $a \leqslant x \leqslant b$, the values of $y$ given by the equation $f(y, x)=0$ are real, these values 
may be represented, to an arbitrarily close approximation, as the values of rational functions of $x$, or the roots of quadratic equations whose coefficients are k.utional functions of $x$; these equations may be obtained by the process described above, the initial divisor being $f_{y}^{\prime}(y, x)$. As a trivial example, if $|x| \leqslant 1$, successive approximations to one value of $y$ given by the equation

$$
y^{8}-3 y^{2}+4 x^{2}=0
$$

are

$$
y=2 x^{2}, \quad y=\left(2 x^{4}-4 x^{2}\right) /\left(2 x^{4}-2 x^{2}-1\right),
$$

and so on. 